Technical note

\title{
Sensing of the time-varying angular rate for MEMS Z-axis gyroscopes
}

\author{
M.H. Salah ${ }^{\text {a }}$, M.L. McIntyre ${ }^{\text {b }}$, D.M. Dawson ${ }^{\text {c }}$, J.R. Wagner ${ }^{\text {d }}$, E. Tatlicioglu ${ }^{\text {e,* }}$ \\ ${ }^{a}$ Department of Mechatronics Engineering, Hashemite University, Zarqa, Jordan \\ ${ }^{\mathrm{b}}$ Department of Engineering, Western Kentucky University, Bowling Green, KY, USA \\ ${ }^{\mathrm{c}}$ Department of Electrical \&' Computer Engineering, Clemson University, Clemson, SC, USA \\ ${ }^{\mathrm{d}}$ Department of Mechanical Engineering, Clemson University, Clemson, SC, USA \\ ${ }^{\mathrm{e}}$ Department of Electrical \& Electronics Engineering, Izmir Institute of Technology, Izmir, Turkey
}

\section{A R T I C L E I N F O}

\section{Article history:}

Received 30 July 2009

Accepted 5 July 2010

\section{Keywords}

MEMS Z-axis gyroscope

Least-squares estimation

Adaptive controller

Adaptive observer

\begin{abstract}
A B S T R A C T
In this paper, a nonlinear estimation strategy for sensing the time-varying angular rate of a Z-axis MEMS gyroscope is presented. An off-line adaptive least-squares estimation strategy is first developed to accurately estimate the unknown model parameters. Both axes of a Z-axis MEMS gyroscope are then actively controlled utilizing an on-line controller/observer to facilitate time-varying angular rate sensing. The proposed nonlinear estimation strategy is developed based on a Lyapunov-based analysis, which proves that the time-varying angular rate experienced by the device can be estimated accurately. Two cases for angular rate are investigated which are time-varying and constant magnitudes. An adaptive controller/observer was also utilized for sensing the angular rate to investigate the performance of the proposed controller/observer. Representative numerical results are discussed to demonstrate the performance of the proposed nonlinear strategy in accurately sensing the applied angular rate. Overall, the proposed nonlinear controller/observer improves sensing the constant angular rate by $50 \%$ and the time-varying angular rate by $90 \%$ when compared with an adaptive controller/observer.
\end{abstract}

(C) 2010 Elsevier Ltd. All rights reserved.

\section{Introduction}

MEMS gyroscopes are often used to measure the angular rate in many applications such as [1] microgravity measurements and platform stabilization in space applications, activity monitoring in biomedical applications, sport equipment in consumer applications, robotics and machine and vibration monitoring in industrial applications, tracking and monitoring mechanical shock and vibration during transportation in automotive applications; and in several military applications such as arming in missiles. The advantage of MEMS gyroscopes over conventional inertial navigation instruments includes features such as [2,3] compact size, decreased weight, reduced power consumption, and low cost micromachining process. Practically all MEMS gyroscopes provide angular rate measurements using vibrational elements; hence, these devices are referred to as vibrational gyroscopes. Previous research has implemented a drive axis/sense axis methodology for angular rate sensing $[4,5]$. Using this method, the gyroscope consists of a mass suspended on elastic flexures anchored to the substrate of the device. This mass is driven at its resonate frequency and the rotation induced Coriolis force generates the transfer of energy from the drive vibrational plane to the sense vibrational plane (i.e., $x-y$ plane in case of the $Z$-axis gyroscope [6]). For MEMS gyro-

\footnotetext{
* Corresponding author. Tel.: +90 232 7506728; fax: +90 2327506599 .

E-mail address: envertatlicioglu@iyte.edu.tr (E. Tatlicioglu).
}

scopes to realize the performance levels of which they are capable, innovative methods are required for device calibration, accurate model identification, and active control of sensor dynamics for angular rate sensing [5].

Past MEMS gyroscope research has focused on the development of the microelectromechanical fabrication processes $[7,8]$, sensor modeling [9], and the active control of the sensor dynamics for model identification and angular rate sensing [10]. M'Closkey et al. [2] presented a dynamic model for the JPL microgyro. This work is extended in [3] with a recursive least-squares algorithm to identify the parameters of the physical model using available sensor information. In [11,12], Lee et al. developed adaptive strategies to a gyro-mirror line-of-sight stabilization platform to estimate the unknown model parameters. Maruyama et al. [13] proposed a gyroscopic sensor using active magnetic bearing to realize high accuracy, compact and low-cost sensors. The authors were able to measure angular velocities and accelerations based on the control signals for cancelling the inertial effects that act on the active magnetic bearing rotor. Heredia et al. [14] presented an actuator and sensor fault detection and isolation system for small autonomous helicopters. The diagnosis of actuator and sensor faults is investigated using a model-based approach, with observer-based residual generation. In [15], two Lyapunov-based adaptive controllers were proposed to compensate for uncertainties in the natural frequencies, mode coupling and damping, however some calibration (i.e., mass parameters of the gyro) was 
required. Recently, in [16-18], control algorithms were designed that reject imperfections (such as disturbances) in MEMS gyroscopes. Shkel et al. [9] proposed both adaptive and non-adaptive nonlinear methods for the active control of the sensor dynamics for angular rate sensing. Their approach compensates for model inaccuracies under the assumption that the angular rate is constant. In fact, most of the relevant past works considered the angular rate to be constant with respect to time $[2,5,10,15,19-21]$ where the angular rate, indeed, is a time-varying signal. Furthermore, on-line estimation of the time-varying angular rate is one of the major control problems associated with MEMS gyroscopes. In the literature, there exists only three past works that considered estimation of time-varying angular rate [22-24] aside from our work. In [22], an adaptive controller for angular rate sensing was developed under the assumption that the time-varying angular rate can be approximated by a polynomial function in a finite time interval. In $[23,24]$, the time-varying angular rate was assumed to be a pure sinusoidal. This is a very restrictive assumption that reduces the application of the estimators designed in $[23,24]$. Our work relaxed the restrictive assumptions of pure sinusoidal timevarying angular rate polynomial function approximated. The estimator designed in our paper observes the time-varying angular rate regardless of its form and it works for any time-varying angular rates. Park and Horowitz in [25] presented a linear adaptive operation strategy for MEMS Z-axis gyroscopes under the assumption of a constant angular rate. This work deviates from the traditional drive axis/sense axis methodology due to a lack of meeting the persistence of excitation condition which is required for compensation of the fabrication defects and perturbations affecting the behavior of a MEMS Z-axis gyroscope. Other research has been presented that develops alternate drive methods for both axes of the $Z$-axis MEMS gyroscope. In [22], the authors extended the previous development of [26] to develop controllers for both axes. Specifically, an adaptive resonant frequency tuning controller for the drive axis was proposed and an adaptive controller for angular rate sensing was developed under the assumption that the timevarying angular rate can be approximated by a polynomial function in a finite time interval.

In this paper, ${ }^{1}$ both axes of the device are actively controlled to develop a strategy for estimating the time-varying angular rate for a Z-axis MEMS gyroscope. An off-line parameter estimation strategy is proposed that places the gyroscope in a condition of zero angular rate. A reference signal excites both axes such that a subsequently required persistence of excitation condition is satisfied. A standard adaptive least-squares estimator is utilized to estimate the unknown model parameters. An analysis is presented which proves that these parameters are accurately estimated. Based on exact knowledge of the model parameters, an on-line active controller/observer is then developed for time-varying angular rate sensing. For this method, a nonlinear algorithm is created based on a Lyapunov-based analysis, which proves that the time-varying angular rate experienced by the device is estimated accurately. The on-line controller/observer performance was compared against an adaptive controller/observer in two cases. For Case 1, the exciting angular rate was constant, while for Case 2 , the angular rate was time-varying. Numerical results are presented which demonstrates the proof of concept of the active control approach.

The rest of this paper is organized as follows. In Section 2, the MEMS gyroscope dynamic model is defined along with the required assumptions for this analysis. In Section 3, the adaptive least-squares estimator is presented with simulation results for the off-line estimation strategy. In Section 4, a nonlinear algorithm is developed with an accompanying analysis to verify that under a

\footnotetext{
${ }^{1}$ A preliminary version of this work has appeared in [27].
}

set of sufficient conditions the time-varying angular rate is accurately estimated. Representative simulation results are presented for the proposed on-line angular rate estimation strategy and adaptive controller/observer is utilized for comparison. Concluding remarks are provided in Section 5.

\section{System dynamics and assumptions}

A Z-axis, non-ideal MEMS gyroscope is depicted in Fig. 1. From this diagram, the dynamic model can be written in Cartesian coordinates as [22]

$M \ddot{q}+D \dot{q}+K q=\tau+S \dot{q}$

where $q(t) \triangleq[x(t), \quad y(t)]^{T} \in \mathbb{R}^{2}$ is the displacement of the gyroscope's reference point, and $x(t), y(t) \in \mathbb{R}$. In (1), $\dot{q}(t), \ddot{q}(t) \in \mathbb{R}^{2}$ represents the velocity and acceleration of the gyroscope's reference point, respectively, $M \in \mathbb{R}^{2 \times 2}$ denotes the inertia effect, $D \in \mathbb{R}^{2 \times 2}$ and $K \in \mathbb{R}^{2 \times 2}$ represent the damping ratio and spring constant, $S(t) \in \mathbb{R}^{2 \times 2}$ denotes the centripetal-Coriolis effect, and $\tau(t) \triangleq$ $\left[\tau_{x}(t), \quad \tau_{y}(t)\right]^{T} \in \mathbb{R}^{2}$ represent the control input with $\tau_{x}(t)$, $\tau_{y}(t) \in \mathbb{R}$.

The terms $M$ and $S(t)$ can be expanded as [22]

$M=\left[\begin{array}{cc}m & 0 \\ 0 & m\end{array}\right], \quad S=\left[\begin{array}{cc}0 & 2 m \Omega_{z} \\ -2 m \Omega_{z} & 0\end{array}\right]$

where $m \in \mathbb{R}$ is the reference point's mass within the gyroscope, and $\Omega_{z}(t) \in \mathbb{R}$ is the time-varying angular rate about the $Z$-axis. The angular rates $\Omega_{x}$ and $\Omega_{y}$ about the $x$ and $y$ axes are assumed to be zero. Due to imperfections in the fabrication process, $D$ and $K$ can be assigned [22] as

$D=\left[\begin{array}{ll}d_{x x} & d_{x y} \\ d_{y x} & d_{y y}\end{array}\right], \quad K=\left[\begin{array}{ll}k_{x x} & k_{x y} \\ k_{y x} & k_{y y}\end{array}\right]$

The terms $d_{x x}, d_{y y} \in \mathbb{R}$ are the damping ratios along the $x$ and $y$ axes, respectively, $d_{x y}, d_{y x} \in \mathbb{R}$ are the damping ratios affecting both $x$ and $y$ axes, $k_{x x}, k_{y y} \in \mathbb{R}$ are the spring constants along the $x$ and $y$ axes, respectively, and $k_{x y}, k_{y x} \in \mathbb{R}$ are the spring constants affecting both $x$ and $y$ axes. To facilitate the off-line parameter and on-line timevarying angular rate estimation strategies, three assumptions frame the analysis.

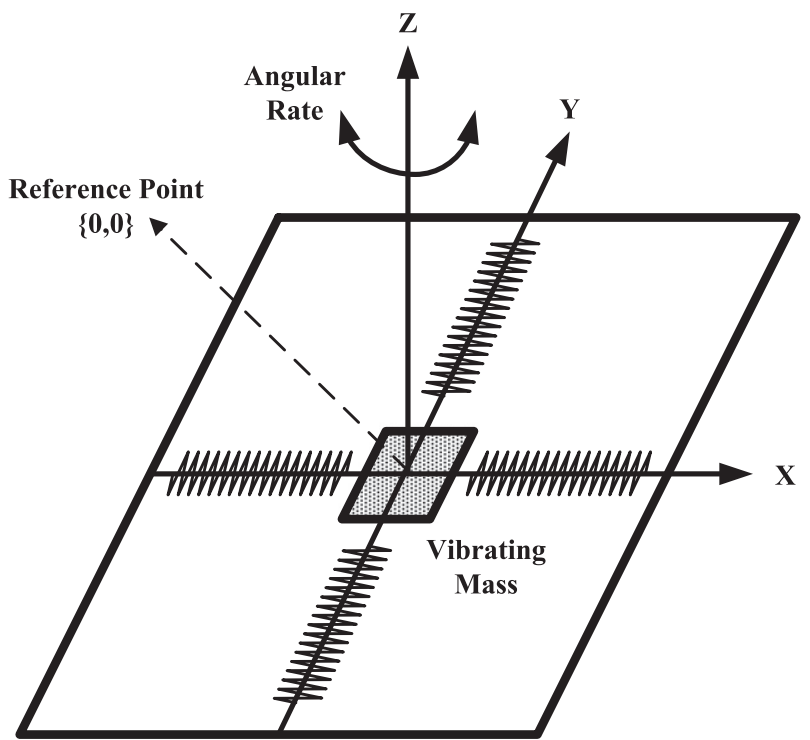

Fig. 1. Mass-spring model of the Z-axis MEMS gyroscope. 
Assumption 1. The gyroscope's parameters $m, d_{x x}, d_{y y}, k_{x x}$, and $k_{y y}$ are unknown and assumed to be constants with respect to time.

Assumption 2. The damping ratios $d_{x y}$ and $d_{y x}$, and the stiffness $k_{x y}$ and $k_{y x}$ are equal to zero. A precedence for this assumption can be found in $[4,8]$. It should be noted that the subsequent development could be extended such that these parameters may also be estimated.

Assumption 3. The time-varying angular rate and its first two time derivatives are bounded, so that $\Omega_{z}(t), \quad \dot{\Omega}_{z}(t), \quad \ddot{\Omega}_{z}(t) \in L_{\infty}$. Hence, $S(t), \quad \dot{S}(t)$, and $\ddot{S}(t)$ are upper bounded as

$\|S(t)\| \leq \Gamma_{1}, \quad\|\dot{S}(t)\| \leq \Gamma_{2}, \quad\|\ddot{S}(t)\| \leq \Gamma_{3}$

where $\Gamma_{1}, \Gamma_{2}, \Gamma_{3} \in \mathbb{R}^{+}$are bounding constants.

\section{Off-line parameter estimation}

The gyroscope system is configured such that the angular rate is equal to zero (i.e., $\Omega_{z}(t)=0$ ). A fourth assumption is imposed such that a reference input is injected at $\tau$ in (1) with $q(t), \quad \dot{q}(t)$ and $\ddot{q}(t)$ bounded.

Assumption 4. The reference input $\tau$ is designed to be a bounded, piecewise continuous function.

With a zero angular rate, the dynamic model in (1) can be rewritten as

$M \ddot{q}+D \dot{q}+K q=\tau$

Since $\ddot{q}(t)$ is unmeasurable, a torque filtering technique [28] may be used. The model in (5) can be expressed as

$\tau=\dot{h}+g$

where $h(t), \quad g(t) \in \mathbb{R}^{2}$ are defined as

$\dot{h} \triangleq \frac{d}{d t}(M \dot{q})=M \ddot{q}, \quad g \triangleq D \dot{q}+K q$

A filtered torque signal $\tau_{f}(t) \in \mathbb{R}^{2}$ can be defined as

$\tau_{f} \triangleq f * \tau$

where $(*)$ is the convolution operator. The variable $f(t) \in \mathbb{R}$ is the impulse response of a linear stable and strictly proper filter that can be defined as a first-order filter with

$f \triangleq \alpha e^{-\beta t}$

where $\alpha, \beta \in \mathbb{R}^{+}$are constants. After substituting (6) into (8) and using standard convolution properties, it may be stated that

$$
\begin{aligned}
\tau_{f} & =f * \dot{h}+f * g \\
& =\dot{f} * h+f_{o} h-f h_{o}+f * g
\end{aligned}
$$

where $f_{o}$ and $h_{o}$ denote that $f(t)$ and $h(t)$ are computed at the initial time $t_{0}$. Now, (8) can be rewritten as

$\tau_{f}=W_{f} \theta$

where $\theta \in \mathbb{R}^{5}$ is a vector of the unknown constant parameters, and $W_{f}(q, \dot{q}) \in \mathbb{R}^{2 \times 5}$ is the known filtered regression matrix which may be expressed as

$\theta \triangleq\left[\begin{array}{lllll}m & d_{x x} & k_{x x} & d_{y y} & k_{y y}\end{array}\right]^{T}$

$W_{f} \triangleq\left[\begin{array}{ccccc}W_{f 11} & W_{f 12} & W_{f 13} & 0 & 0 \\ W_{f 21} & 0 & 0 & W_{f 24} & W_{f 25}\end{array}\right]$
The elements of the regression matrix, $W_{f}(q, \dot{q})$, may be generated using the expressions

$$
\begin{aligned}
& W_{f 11} \triangleq \alpha \dot{x}+p_{x}+\alpha e^{-\beta t_{o}} \dot{x}-\alpha e^{-\beta t} \dot{x}_{o} \\
& W_{f 12} \triangleq-\beta W_{f 12}+\alpha \dot{x} \\
& W_{f 13} \triangleq-\beta W_{f 13}+\alpha x \\
& W_{f 21} \triangleq \alpha \dot{y}+p_{y}+\alpha e^{-\beta t_{o}} \dot{y}-\alpha e^{-\beta t} \dot{y}_{o} \\
& W_{f 24} \triangleq-\beta W_{f 24}+\alpha \dot{y} \\
& W_{f 25} \triangleq-\beta W_{f 25}+\alpha y
\end{aligned}
$$

where $\alpha$ and $\beta$ were introduced in (9). The terms $W_{f 11}(t), W_{f 12}(t), W_{f 13}(t), W_{f 21}(t), W_{f 24}(t) W_{f 25}(t) \in \mathbb{R}$ and $\dot{x}_{o}$ and $\dot{y}_{o}$ denote that $\dot{x}(t)$ and $\dot{y}(t)$ are computed at the initial time $t_{0}$. The variables $p_{x}(t), p_{y}(t) \in \mathbb{R}$ are auxiliary filter signals which are defined as $\dot{p}_{x}=-\beta p_{x}-\alpha \beta \dot{x}, \quad \dot{p}_{y}=-\beta p_{y}-\alpha \beta \dot{y}$

Further, $\quad W_{f 12}\left(t_{o}\right)=W_{f 13}\left(t_{o}\right)=W_{f 24}\left(t_{o}\right)=W_{f 25}\left(t_{o}\right)=p_{x}\left(t_{o}\right)=p_{y}\left(t_{o}\right)=0$. Since $\theta$ is a vector of uncertain parameters, the structure of (11) cannot be implemented. An implementable form (i.e., a measurable, acceleration independent form) of (11) can be determined by utilizing (8) and (9) to generate the filtered torque signal so that

$\dot{\tau}_{f}=-\beta \tau_{f}+\alpha \tau, \quad \tau_{f}\left(t_{o}\right)=\emptyset_{2}$

where $\emptyset_{2} \in \mathbb{R}^{2}=\left[\begin{array}{ll}0, & 0\end{array}\right]^{T}$ is a vector of zeros.

Lemma 1. The parameterized model described by (11)-(14) is equal to the filtered torque dynamics described by (15).

Proof. See Appendix A in [29].

Let the estimate of the filtered torque $\hat{\tau}_{f}(t) \in \mathbb{R}^{2}$ be defined as $\hat{\tau}_{f} \triangleq W_{f} \hat{\theta}$

where $\hat{\theta}(t) \in \mathbb{R}^{5}$ is the estimate of the unknown parameters, and $W_{f}(q, \dot{q})$ is defined in (12)-(14). An error signal $\varepsilon(t) \in \mathbb{R}^{2}$ can also be defined as

$\varepsilon \triangleq \tau_{f}-\hat{\tau}_{f}=W_{f} \tilde{\theta}$

The parameter estimate error $\tilde{\theta}(t) \in \mathbb{R}^{5}$ is defined as

$\tilde{\theta} \triangleq \theta-\hat{\theta}$

An adaptive update rule can be generated using a least-squares estimation method [28]

$\dot{\hat{\theta}} \triangleq=k P W_{f}^{T} \varepsilon$

where $k \in \mathbb{R}^{+}$is a constant, and $P(t) \in \mathbb{R}^{5 \times 5}$ is the covariance matrix. This matrix $P(t)$ is generated by the covariance propagation equation

$\dot{P} \triangleq=-k P W_{f}^{T} W_{f} P$

Theorem 1. The least-squares estimation strategy, described in (19) and (20), ensures that $\tilde{\theta}(t) \rightarrow 0$ as $t \rightarrow \infty$ provided the following sufficient conditions are meet: (i) The plant of estimation is strictly proper, (ii) the input is piecewise continuous and bounded, (iii) the output of the plant of estimation is bounded, and the following persistence of excitation condition [30] holds

$\gamma_{1} I_{5} \leq \int_{t}^{t+\delta} W_{f}^{T}(\sigma) W_{f}(\sigma) d \sigma \leq \gamma_{2} I_{5}$

where $\gamma_{1}, \gamma_{2} \in \mathbb{R}^{+}$are constants, $I_{5} \in \mathbb{R}^{5 \times 5}$ is the identity matrix, and $W_{f}(\cdot)$ is defined in (12)-(14). 
Proof. To prove that $\tilde{\theta}(t) \rightarrow 0$ as $t \rightarrow \infty$, Theorem 2.5.3 from [30] is applied. To prove that sufficient conditions (i)-(iii) are valid; the plant of estimation described in (15) is strictly proper, the reference input (i.e., $\tau$ ) to the plant is designed such that it is piecewise continuous and bounded, and $\tau$ has been designed to be bounded, then from standard linear analysis tools, (15) can be used to show that $\tau_{f}(t), \dot{\tau}_{f}(t) \in L_{\infty}$.

\subsection{Numerical results for the parameter estimation}

A numerical simulation was created to demonstrate the performance of the least-squares estimator given in (19) and (20) for a $Z$ axis MEMS gyroscope with dynamics in (5). A reference input was selected to drive both axes to meet the persistently excitation condition of (21) with

$$
\begin{aligned}
\tau_{x}= & \sin \left(\frac{2}{5} \pi t\right)+\frac{1}{9.6} \sin \left(\frac{10.4}{5} \pi t\right)+\frac{1}{25.5} \sin \left(\frac{27.4}{5} \pi t\right) \\
& +\frac{1}{59.12} \sin \left(\frac{52.2}{5} \pi t\right) \\
\tau_{y}= & 2 \sin \left(\frac{2}{7} \pi t\right)+\frac{2}{7.6} \sin \left(\frac{10.4}{7} \pi t\right)+\frac{2}{22.5} \sin \left(\frac{37.4}{7} \pi t\right) \\
& +\frac{2}{55.12} \sin \left(\frac{44.2}{7} \pi t\right)
\end{aligned}
$$

The gyroscope's parameters and initial values were $d_{x x}=1.2 \mu \mathrm{N} \mathrm{s} /$ $\mathrm{mm}, d_{y y}=1.5 \mu \mathrm{N} \mathrm{s} / \mathrm{mm}, k_{x x}=45 \mu \mathrm{N} / \mathrm{mm}, k_{y y}=40 \mu \mathrm{N} / \mathrm{mm}, \mathrm{m}=1 \mathrm{~g}$, $P\left(t_{o}\right)=\operatorname{diag}\{125, \quad 125, \quad 125, \quad 125, \quad 125\}, \quad q\left(t_{0}\right)=\dot{q}\left(t_{0}\right)=\ddot{q}\left(t_{0}\right)=$ $\left[\begin{array}{ll}0, & 0\end{array}\right]^{T}, \hat{\theta}\left(t_{o}\right)=\left[\begin{array}{lllll}2.1, & 0.3, & 20, & 2.6, & 63\end{array}\right]^{T}$, where $t_{o}=0$. The estimator gains were set to be $\alpha=\beta=1$ and $k=50$. The estimated values for the mass, damping ratios, and the spring constants are presented in Fig. 2. The simulation data shows that all of the parameter estimates are within $\pm 0.3 \%$ of their actual values after $t=75 \mathrm{~s}$.

\section{On-line time-varying angular rate estimator design}

A global asymptotic result will exist provided that the displacement $q(t)$ and velocity $\dot{q}(t)$ of the gyroscope's reference point are measurable, and the model parameters defined in (12) are known a priori. This development requires that the control input $\tau$, introduced in (1), is designed to force $q(t)$ to track a given known trajectory $q_{k}(t) \triangleq\left[x_{k}(t), \quad y_{k}(t)\right]^{T} \in \mathbb{R}^{2}$ to estimate $\Omega_{z}$, where $x_{k}(t), y_{k}(t) \in \mathbb{R}$. Further, the given known trajectory and its first three time derivatives are bounded (i.e., $q_{k}(t), \dot{q}_{k}(t), \ddot{q}_{k}(t)$, $\left.\dddot{q}_{k}(t) \in L_{\infty}\right)$.

\subsection{Estimator design objectives}

The control objective of the angular rate estimator ensures that the reference point's displacement $q(t)$ tracks the given known trajectory $q_{k}$ with

$q(t) \rightarrow q_{k} \quad$ as $\quad t \rightarrow \infty$

The estimator objective requires the estimated angular rate, $\widehat{\Omega}_{z}(t) \in \mathbb{R}$, to converge to the actual rate as

$\widehat{\Omega}_{z}(t) \rightarrow \Omega_{z}$ as $t \rightarrow \infty$

The control objective is achieved utilizing a filtered tracking error signal, $r(t) \in \mathbb{R}^{2}$, defined as

$r \triangleq \dot{e}_{2}+\alpha_{2} e_{2}$

where $e_{2}(t) \in \mathbb{R}^{2}$ is defined as

$e_{2} \triangleq \dot{e}_{1}+\alpha_{1} e_{1}$ with control gains $\alpha_{1}, \alpha_{2} \in \mathbb{R}^{+}$. The error signal, $e_{1}(t) \in \mathbb{R}^{2}$, is defined as

$e_{1} \triangleq q_{k}-q$

Based on the definition of $e_{1}$ in (28), if $e_{1}(t) \rightarrow 0$ as $t \rightarrow \infty$, then $q(t) \rightarrow q_{k}$ as $t \rightarrow \infty$; thus meeting the control objective.

\subsection{Closed-loop error system development}

The second time derivative of (28) may be computed and both sides multiplied by $M$ to obtain

$M \ddot{e}_{1}=M \ddot{q}_{k}+D \dot{q}+K q-S \dot{q}-\tau$

where (1) was utilized. The control input $\tau$ is designed as

$\tau \triangleq M \ddot{q}_{k}+D \dot{q}+K q-\widehat{F}$

with user specified auxiliary signal, $\widehat{F} \in \mathbb{R}^{2}$. After substituting (30) into (29), the expression for the second time derivative of error signal, $\ddot{e}_{1}$, becomes

$M \ddot{e}_{1}=\widehat{F}-S \dot{q}$

From the definition in (27) and utilizing (31), an expression for $\ddot{e}_{2}$ can be written as

$M \ddot{e}_{2}=\dot{\widehat{F}}-\dot{S} \dot{q}-S \ddot{q}+\alpha_{1} M \ddot{e}_{1}$

The closed-loop error system dynamics can now be stated as

$M \dot{r}=\dot{\widehat{F}}-\dot{S} \dot{q}-S \ddot{q}+\alpha_{1} M \ddot{e}_{1}+\alpha_{2} M \dot{e}_{2}$

where (26) was pre-multiplied by $M$ and (32) was substituted. The resulting expression may be rewritten as

$M \dot{r}=\widetilde{N}+N_{k}+\dot{\widehat{F}}-e_{2}$

where the auxiliary signal $\widetilde{N}(q, \dot{q}, \ddot{q}, t) \in \mathbb{R}^{2}$ is defined as

$\widetilde{N} \triangleq N-N_{k}$

where the terms $N(q, \dot{q}, \ddot{q}, t) \in \mathbb{R}^{2}$ and $N_{k}(t) \in \mathbb{R}^{2}$ are defined as

$N \triangleq-\dot{S} \dot{q}-S \ddot{q}+\alpha_{1} M \ddot{e}_{1}+\alpha_{2} M \dot{e}_{2}+e_{2}$

$\left.N_{k} \triangleq N\right|_{q=q_{k}, \dot{q}=\dot{q}_{k}, \ddot{q}=\ddot{q}_{k}}=-\dot{S} \dot{q}_{k}-S \ddot{q}_{k}$

Based on (34), the auxiliary signal introduced in (30) is designed as $\widehat{F}=-\left(k_{s}+1\right)\left[e_{2}(t)-e_{2}\left(t_{0}\right)+\alpha_{2} \int_{t_{0}}^{t} e_{2}(\sigma) d \sigma\right]-\beta_{1} \int_{t_{0}}^{t} \operatorname{sgn}\left(e_{2}(\sigma)\right) d \sigma$

where $k_{s}$ and $\beta_{1}$ are control gains, $t_{o}$ is the initial time, and $\operatorname{sgn}(\cdot) \in \mathbb{R}^{2}$ denotes the vector signum function. The term $e_{2}\left(t_{o}\right)$ in (38) is included so that $\widehat{F}\left(t_{0}\right)=\emptyset_{2}$. The time derivative of the auxiliary signal in (38) may be stated as

$\dot{\hat{F}}=-\left(k_{s}+1\right) r-\beta_{1} \operatorname{sgn}\left(e_{2}\right)$

After substituting (39) into (34), the closed-loop error system becomes

$M \dot{r}=\widetilde{N}+N_{k}-\left(k_{s}+1\right) r-\beta_{1} \operatorname{sgn}\left(e_{2}\right)-e_{2}$

Remark 1. Using the expression in (37), and the fact that $q_{k}(t), \quad \dot{q}_{k}(t), \quad \ddot{q}_{k}(t), \quad \ddot{q}_{k}(t) \in L_{\infty}$, terms $\left\|N_{k}(t)\right\|$ and $\left\|\dot{N}_{k}(t)\right\|$ can be upper bounded by known positive constants $\varsigma_{1}, \quad \varsigma_{2} \in \mathbb{R}$ as

$\left\|N_{k}(t)\right\| \leq \varsigma_{1}, \quad\left\|\dot{N}_{k}(t)\right\| \leq \varsigma_{2}$ 

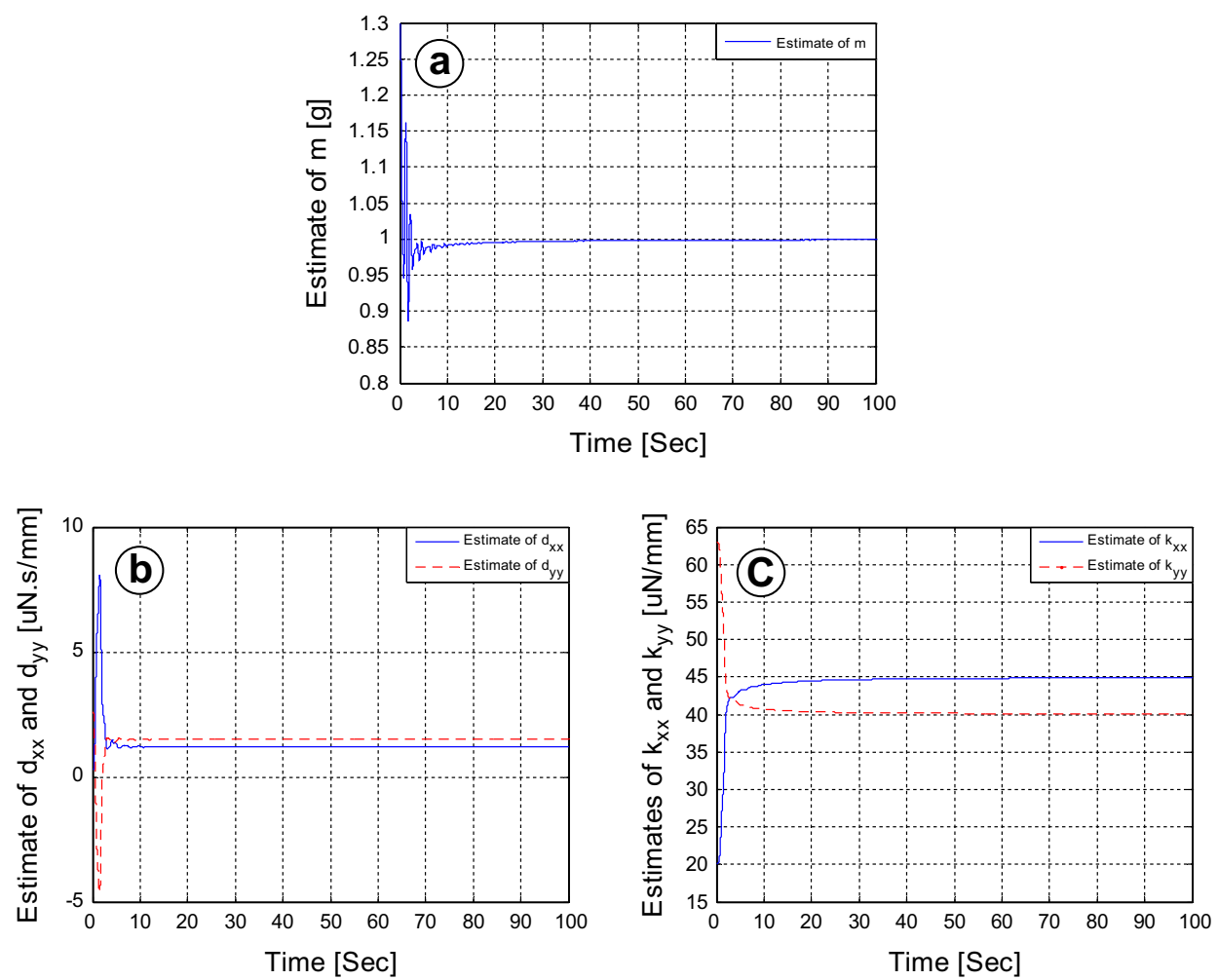

Fig. 2. System parameter estimates: (a) mass, $\hat{m}$; (b) damping ratios, $\hat{d}_{x x}$ and $\hat{d}_{y y}$; and (c) spring constants, $\hat{k}_{x x}$ and $\hat{k}_{y y}$

\subsection{Stability analysis}

Theorem 2. The controller, given in (30) and (38), ensures that $\left\|e_{1}(t)\right\|,\left\|\dot{e}_{1}(t)\right\|,\left\|\ddot{e}_{1}(t)\right\| \rightarrow 0$ as $t \rightarrow \infty$ and all closed-loop signals are bounded provided the control gain $\beta_{1}$, introduced in (38), is selected to satisfy the following sufficient condition

$\beta_{1}>\varsigma_{1}+\frac{1}{\alpha_{2}} \varsigma_{2}$

where the parameters $\varsigma_{1}$ and $\varsigma_{2}$ are bounding constants as shown in (41).

Proof. See Appendix 2 in [29].

\subsection{Time-varying angular rate estimation}

The expression in (31) can be rewritten as

$\widehat{F}-S \dot{q}_{k}=M \ddot{e}_{1}-S \dot{e}_{1}$

where the time derivative of (28) was utilized. Let the error signal $\widetilde{F}(t) \in \mathbb{R}^{2}$ be defined as

$\widetilde{F}=\widehat{F}-F \Rightarrow\left[\begin{array}{l}\widetilde{F}_{1} \\ \widetilde{F}_{2}\end{array}\right]=\left[\begin{array}{l}\widehat{F}_{1}-F_{1} \\ \widehat{F}_{2}-F_{2}\end{array}\right]=M \ddot{e}_{1}-S \dot{e}_{1}$

where $F_{1}(t), \quad F_{2}(t), \quad \widehat{F}_{1}(t), \quad \widehat{F}_{2}(t), \quad \widetilde{F}_{1}(t), \quad \widetilde{F}_{2}(t) \in \mathbb{R}$. From the proof of Theorem 2, $\left\|e_{1}(t)\right\|,\left\|\dot{e}_{1}(t)\right\|,\left\|\ddot{e}_{1}(t)\right\| \rightarrow 0$ as $t \rightarrow \infty$. Hence, $\widetilde{F}(t) \rightarrow 0$ as $t \rightarrow \infty$ so that $\widehat{F}(t) \rightarrow F(t)$ as $t \rightarrow \infty$. The elements of $F(t)$ can be defined as

$F_{1} \triangleq 2 m \Omega_{z} \dot{y}_{k}, \quad F_{2} \triangleq-2 m \Omega_{z} \dot{x}_{k}$

If the auxiliary function $\Delta(t) \in \mathbb{R}^{2}$ is defined as

$\Delta \triangleq-\dot{x}_{k} F_{2}+\dot{y}_{k} F_{1}$ then the expression in (46) can be rewritten as

$\Delta \triangleq 2 m \Omega_{z}\left(\dot{x}_{k}^{2}+\dot{y}_{k}^{2}\right)$

where (45) was utilized. From (46) and (47), an expression for the time-varying angular rate $\Omega_{z}$ can be defined as

$\Omega_{z} \triangleq \frac{\Delta}{2 m\left(\dot{x}_{k}^{2}+\dot{y}_{k}^{2}\right)}=\frac{\dot{y}_{k} F_{1}-\dot{x}_{k} F_{2}}{2 m\left(\dot{x}_{k}^{2}+\dot{y}_{k}^{2}\right)}$

Remark 2. Special care must be taken to avoid $\left(\dot{x}_{k}^{2}(t)+\dot{y}_{k}^{2}(t)\right)=0$ in (48). To avoid this condition, $\dot{x}_{k}$ and $\dot{y}_{k}$ must be designed such that $\left(\dot{x}_{k}^{2}(t)+\dot{y}_{k}^{2}(t)\right)$ is never equal to zero.

Based on (48), the observed time-varying angular rate $\widehat{\Omega}_{z}(t) \in \mathbb{R}$ can be written as

$\widehat{\Omega}_{z}=\frac{\dot{y}_{k} \widehat{F}_{1}-\dot{x}_{k} \widehat{F}_{2}}{2 m\left(\dot{x}_{k}^{2}+\dot{y}_{k}^{2}\right)}$

where $\widehat{F}_{1}$ and $\widehat{F}_{2}$ are generated by (38). An angular rate error signal $\widehat{\Omega}_{z}(t) \in \mathbb{R}$ can be calculated from (48) and (49) as

$\widetilde{\Omega}_{z} \triangleq \widehat{\Omega}_{z}-\Omega_{z}=\frac{\dot{y}_{k} \widetilde{F}_{1}-\dot{x}_{k} \widetilde{F}_{2}}{2 m\left(\dot{x}_{k}^{2}+\dot{y}_{k}^{2}\right)}$

From (50), since $\widetilde{F}_{1}(t), \widetilde{F}_{2}(t) \rightarrow 0$ as $t \rightarrow \infty$, then $\widetilde{\Omega}_{z}(t) \rightarrow 0$ as $t \rightarrow \infty$. Hence, $\widehat{\Omega}_{z}(t) \rightarrow \Omega_{z}$ as $t \rightarrow \infty$.

\subsection{Numerical results for angular rate controller/observer}

An off-line least-squares estimation strategy was performed to estimate the gyroscope's unknown parameters in Section 3. These parameter estimates were used in (1) to demonstrate the on-line nonlinear controller/observer performance per (30) and (38) with exact model knowledge. The on-line controller/observer perfor- 
mance was compared against an adaptive controller/observer (refer to Appendix A) in two cases. For Case 1, the exciting angular rate was constant, $1 \mathrm{rad} / \mathrm{s}$, while for Case 2 , the angular rate was time-varying, $\Omega_{z}=0.5(\sin (t)+\sin (2 t)+\sin (3 t)+\sin (4 t))$. The control input $\tau$ in (30) was modified as

$\tau \triangleq \widehat{M} \ddot{q}_{k}+\widehat{D} \dot{q}+\widehat{K} q-\widehat{F}$

where $\widehat{M}, \widehat{D}$ and $\widehat{K}$ are generated using the off-line estimation strategy. To satisfy Remark 2, consider the given known trajectory for the tracking problem

$\left[\begin{array}{l}x_{k} \\ y_{k}\end{array}\right]=\left[\begin{array}{l}0.5 \sin (\pi t) \\ 0.5 \cos (\pi t)\end{array}\right]$

The gyroscope's parameter estimates, obtained from the leastsquares estimator, were utilized and the control/estimator gains were $\alpha_{1}=10, \alpha_{2}=2, \beta_{1}=2$, and $k_{s}=1000$. The estimated angular
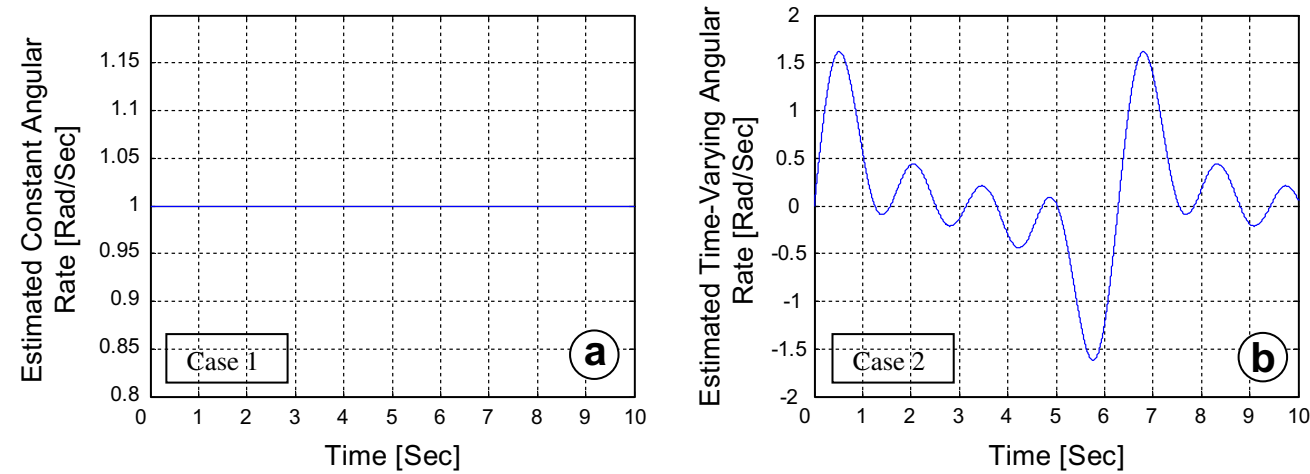

Fig. 3. Estimated angular rate: (a) Case 1: constant magnitude and (b) Case 2: time-varying magnitude.
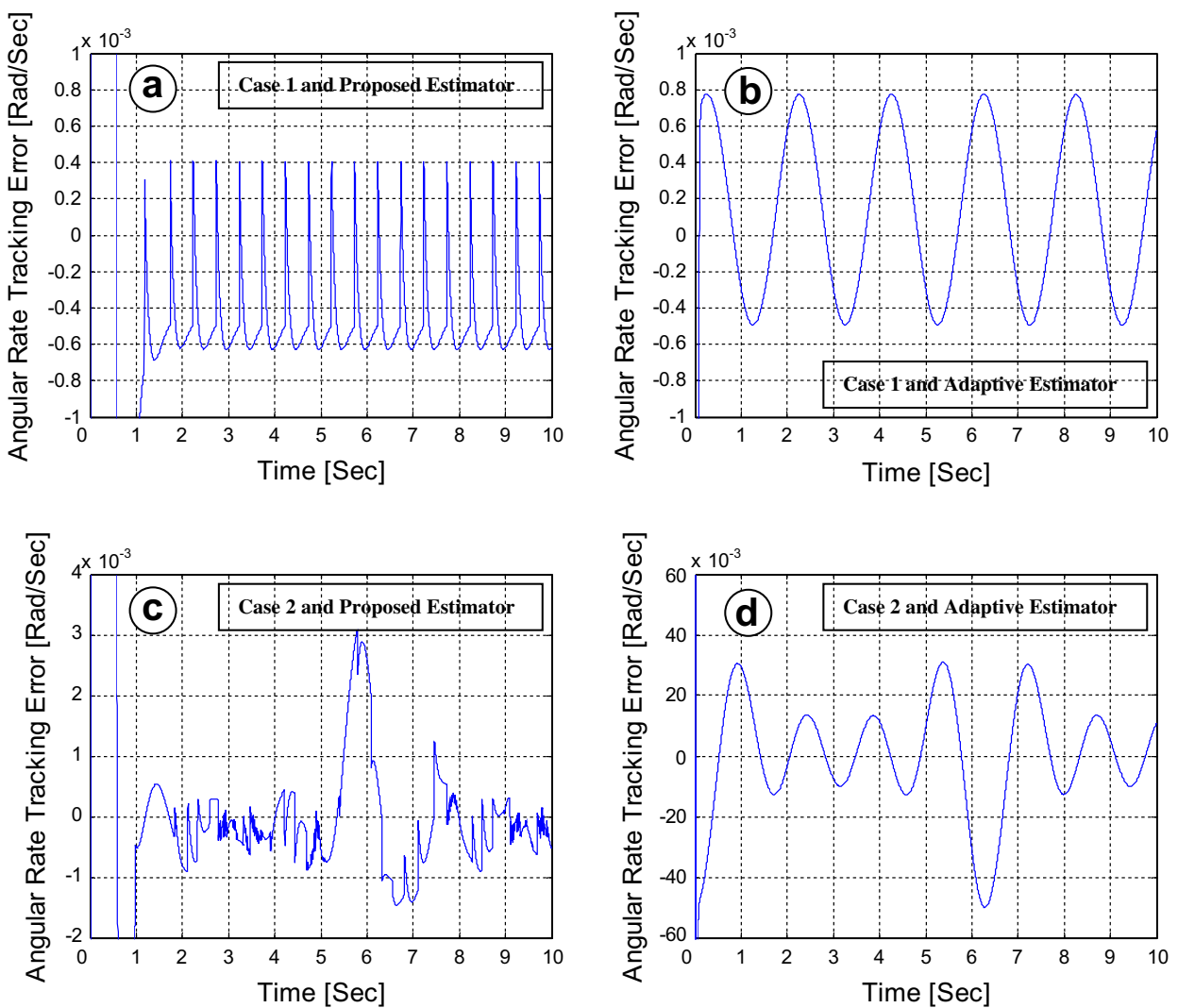

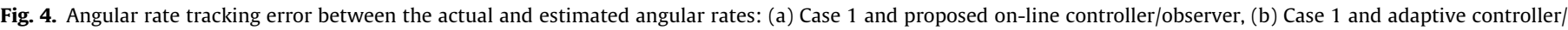
observer, (c) Case 2 and proposed on-line controller/observer, and (d) Case 2 and adaptive controller/observer 
time-varying angular rate sensing nonlinear algorithm is developed based on Lyapunov-based analysis. This on-line nonlinear algorithm required that model parameters be known a priori. Simulation results were presented to illustrate the feasibility of fusing the off-line parameter estimation and the on-line time-varying angular rate sensing controller/observer. The proposed on-line time-varying angular rate sensing nonlinear controller/observer was compared with an adaptive controller/observer and showed significantly better performance in sensing the angular rate.

\section{Appendix A. Adaptive controller/observer}

The development of the adaptive estimator is based on the assumption that the angular rate is constant all times. The subsequent analysis helps estimate the constant angular rate, $\Omega_{z}$ and offers a global asymptotic result provided that the displacement $q(t)$ and velocity $\dot{q}(t)$ of the gyroscope's reference point are measurable. Further, the model parameters defined in (12) must be known $a$ priori. This development requires that the given known trajectory and its first two time derivatives to be bounded; hence, it is assumed that $q_{k}(t), \quad \dot{q}_{k}(t), \quad \ddot{q}_{k}(t) \in L_{\infty}$.

Let the filtered tracking error signal, denoted by $s(t) \in \mathbb{R}^{2}$, be defined as

$s \triangleq=\dot{e}+\alpha e$

where $\alpha \in \mathbb{R}^{+}$is the control gain. The error, $e(t) \in \mathbb{R}^{2}$, may be defined as

$e \triangleq q_{k}-q$

Based on the definition of $e(t)$ in (A.2), if $e(t) \rightarrow 0$ as $t \rightarrow \infty$, then $q(t) \rightarrow q_{k}$ as $t \rightarrow \infty$, thus, meeting the control objective. To develop the closed-loop error system for $s(t)$, the second time derivative of (A.2) is taken and then both sides are multiplied by $M$ to obtain

$M \ddot{e}=M \ddot{q}_{k}+D \dot{q}+K q-\tau-W \Omega_{z}$

where (1) was utilized. The term $S \dot{q}$ in (1) was parameterized as

$S \dot{q}=\left[\begin{array}{cc}2 m \Omega_{z} & 0 \\ 0 & -2 m \Omega_{z}\end{array}\right]\left[\begin{array}{c}\dot{x} \\ \dot{y}\end{array}\right]=\left[\begin{array}{c}2 m \Omega_{z} \dot{x} \\ -2 m \Omega_{z} \dot{y}\end{array}\right]=\left[\begin{array}{c}2 m \dot{x} \\ -2 m \dot{y}\end{array}\right] \Omega_{z} \triangleq W \Omega_{z}$

The control input $\tau(t)$ is designed as

$\tau \triangleq M \ddot{q}_{k}+D \dot{q}+K q-\widehat{F}$

where $\widehat{F} \in \mathbb{R}^{2}$ is an auxiliary signal to be subsequently selected. After substituting (A.5) into (A.3), the following expression can be obtained

$M \ddot{e}=\widehat{F}-W \Omega_{z}$

Utilizing (A.1) and (A.6), the closed-loop error system dynamics can be written as

$M \dot{s}=\widehat{F}-W \Omega_{z}+\alpha M \dot{e}$

Based on (A.7), the auxiliary signal introduced in (A.5) is designed as

$\widehat{F}=W \widehat{\Omega}_{z}-\left(k_{1}+k_{2}\right) s$

where $k_{1}, k_{2} \in \mathbb{R}^{+}$are control gains and $\widehat{\Omega}_{z} \in \mathbb{R}$ is the estimate of the constant angular rate. After substituting (A.8) into (A.7), the closedloop error system becomes

$M \dot{s}=W \widetilde{\Omega}_{z}-\left(k_{1}+k_{2}\right) s+\alpha M \dot{e}$

where $\widetilde{\Omega}_{z}=\widehat{\Omega}_{z}-\Omega_{z}$. An update law to estimate the angular rate, $\widehat{\Omega}_{z}$, can be specified as

$\dot{\widehat{\Omega}}_{z}=\left(k_{3}-W\right)^{T} s-k_{4} \widetilde{\Omega}_{z}$ where $k_{3} \triangleq\left[k_{31}, \quad k_{32}\right]^{T} \in \mathbb{R}^{2}$ and $k_{31}, k_{32}, k_{4} \in \mathbb{R}^{+}$are the control gains.

A Lyapunov-based stability analysis is performed to prove that the constant angular rate, $\Omega_{z}$, can be estimated accurately. Utilizing the Lyapunov function $V \triangleq \frac{1}{2} e^{T} e+\frac{1}{2} r^{T} M r+\frac{1}{2} \widetilde{\Omega}_{z}^{2}$, the inequality $\dot{V} \leqslant-\gamma\|z\|^{2}$ can be obtained. In this instance, $\gamma=\lambda_{3}-\frac{\rho^{2}}{4 k_{2}}, \lambda_{3}=$ $\min \left\{\alpha, \quad k_{1}, \quad k_{4}\right\}$, and $z \triangleq\left[e, \quad s, \quad \widetilde{\Omega}_{z}\right]^{T} \in \mathbb{R}^{3}$ where $\lambda_{3} \geqslant \frac{\rho^{2}}{4 k_{2}}$. Finally, similar argument as Appendix 2 in [29] can show that all signals are bounded and $e(t), \dot{e}(t), s(t), \widetilde{\Omega}_{z}(t) \rightarrow 0$ as $t \rightarrow \infty$; thus, $\widehat{\Omega}_{z}(t) \rightarrow \Omega_{z}$ as $t \rightarrow \infty$.

\section{References}

[1] Yazdi N, Ayazi F, Najafi K. Micromachined inertial sensors. Proc IEEE 1998;86(8):1640-59.

[2] M'Closkey R, Gibson S, Hui J. Model parameter identification of a MEMS gyroscope. In: Proceedings of the American control conference; June 2000. p. 1699-704.

[3] M'Closkey R, Gibson S, Hui J. Input-output dynamics of the JPL microgyroscope. In: Proceedings of the 37th IEEE conference on decision and control; December 1998. p. 4328-33.

[4] Pagilla P, Zhu Y. Adaptive control of mechanical systems with time-varying parameters and disturbances. J Dyn Syst Meas Control 2004;126(3):520-30.

[5] Shkel A, Horowitz R, Seshia A, Park S, Howe R. Dynamics and control of micromachined gyroscopes. In: Proceedins of the American control conference; June 1999. p. 2119-24

[6] Clark W, Howe R, Horowitz R. Surface micromachined Z-axis vibratory rate gyroscope. In: Proceedings of IEEE solid state sensors and actuators workshop; June 1996. p. 283-7.

[7] Tang T, Gutierrez R, Stell C, Vorperian V, Arakaki G, Rice J, et al. A packaged silicon MEMS vibratory gyroscope for microspacecraft. In: Proceedings of IEEE of 10th annual international workshop on MEMS; January 1997. p. 500-5.

[8] Tang T, Gutierrez R, Wilcox J, Stell C, Vorperian V, Dickerson M, et al. Silicon bulk micromachined vibratory gyroscope for microspacecraft. In: Proceedings of SPIE of International Society of Optical Engineering; August 1996. p. 101-15.

[9] Shkel A, Howe R, Horowitz R. Modeling and simulation of micromachined gyroscopes in the presence of imperfections. In: Proceedings of the international conference on modeling and simulation of microsystems; April 1999. p. 605-8.

[10] Park S, Horowitz R. Adaptive control for the conventional mode of operation of MEMS gyroscopes. J Microelectromech Syst 2003;12(1):101-8.

[11] Lee T, Tan K, Lee M. A variable-structure augmented adaptive controller for a gyro-mirror line-of-sight stabilization platform. Mechatronics 1998;8(1): 47-64.

[12] Lee T, Ge S, Wong C. Adaptive neural network feedback control of a passive line-of-sight stabilization system. Mechatronics 1998;8(8):887-903.

[13] Maruyama Y, Mizuno T, Takasaki M, Ishino Y, Kameno H. Extension of measurement bandwidth in an AMB-based gyroscopic sensor. Mechatronics 2009;19(8):1261-8.

[14] Heredia G, Ollero A, Mejar B, Mahtani R. Sensor and actuator fault detection in small autonomous helicopters. Mechatronics 2008;18(2):90-9.

[15] Leland R. Adaptive control of a MEMS gyroscope using Lyapunov methods IEEE Trans Control Syst Technol 2006;14(2):278-83.

[16] Park S, Horowitz R, Tan C-W. Dynamics and control of a MEMS angle measuring gyroscope. Sensors Actuat A: Phys 2008;144(1):56-63.

[17] Fast B, Miklosovic R, Radke A. Active disturbance rejection control of a MEMS gyroscope. In: Proceedings of the American control conference; June 2008. p. 3746-50.

[18] Zheng Q Dong L, Lee D, Gao Z. Active disturbance rejection control for MEMS gyroscopes. In: Proceedings of the American control conference; June 2008. p. 4425-30.

[19] Leland R, Lipkin Y, Highsmith A. Adaptive oscillator control for a vibrational gyroscope. In: Proceedings of the American control conference; July 2003. p. 3347-52.

[20] Park S, Adaptive control strategies for MEMS gyroscopes, PhD dissertation, The University of California Berkeley; 2000.

[21] John J, Vinay T. Novel concept of a single-mass adaptively controlled triaxial angular rate sensor. IEEE Sensors J 2006;6(3):588-95.

[22] Dong L, Leland R. The adaptive control system of a MEMS gyroscope with timevarying rotation rate. In: Proceedings of the American control conference; June 2005. p. 3592-7.

[23] Zheng Q. On active disturbance rejection control: stability analysis and applications in disturbance decoupling control, PhD dissertation, Cleveland State University; 2009.

[24] Tsai N-C, Sue C-Y. Integrated model reference adaptive control and timevarying angular rate estimation for micro-machined gyroscopes. Int J Control 2010;83(2):246-56

[25] Park S, Horowitz R. New adaptive mode of operation for MEMS gyroscopes. ASME J Dyn Syst Meas Control 2004;126(4):800-10. 
[26] Leland R. Adaptive mode tuning for vibrational gyroscopes. IEEE Trans Control Syst Technol 2003;11(2):242-7.

[27] Salah M, McIntyre M, Dawson D, Wagner J. Time-varying angular rate sensing for a MEMS Z-axis gyroscope. In: Proceedings of the IEEE conference on decision and control; December 2006. p. 2165-70.

[28] Lewis F, Dawson D, Abdallah C. Robot manipulator control: theory and practice. second ed. New York: Marcel Dekker Inc.; 2004.
[29] Salah M, McIntyre M, Dawson D, Wagner J. Time-varying angular rate sensing for a MEMS Z-axis gyroscope, Clemson University CRB Technical Report, CU/CRB/2/24/06/\#1. <http://www.ces.clemson.edu/ece/crb/publictn/ tr.htm>.

[30] Sastry S, Bodsom M. Adaptive control: stability, convergence and robustness. New Jersey: Prentice Hall Inc.; 1989. 